\title{
Neural Dissociation between Visual Awareness and Spatial Attention
}

\author{
Valentin Wyart ${ }^{1,2}$ and Catherine Tallon-Baudry ${ }^{1,2,3}$ \\ ${ }^{1}$ Université Pierre et Marie Curie-Paris 6, 75005 Paris, France, ${ }^{2}$ Centre National de la Recherche Scientifique, Cognitive Neuroscience and Brain Imaging \\ Laboratory, Unité Propre de Recherche 640, 75013 Paris, France, and ${ }^{3}$ Magneto- and Electroencephalography Center, Hôpital de la Pitié-Salpêtrière, 75013 \\ Paris, France
}

To what extent does what we consciously see depend on where we attend to? Psychologists have long stressed the tight relationship between visual awareness and spatial attention at the behavioral level. However, the amount of overlap between their neural correlates remains a matter of debate. We recorded magnetoencephalographic signals while human subjects attended toward or away from faint stimuli that were reported as consciously seen only half of the time. Visually identical stimuli could thus be attended or not and consciously seen or not. Although attended stimuli were consciously seen slightly more often than unattended ones, the factorial analysis of stimulus-induced oscillatory brain activity revealed distinct and independent neural correlates of visual awareness and spatial attention at different frequencies in the gamma range $(30-150 \mathrm{~Hz})$. Whether attended or not, consciously seen stimuli induced increased mid-frequency gamma-band activity over the contralateral visual cortex, whereas spatial attention modulated high-frequency gammaband activity in response to both consciously seen and unseen stimuli. A parametric analysis of the data at the single-trial level confirmed that the awareness-related mid-frequency activity drove the seen-unseen decision but also revealed a small influence of the attentionrelated high-frequency activity on the decision. These results suggest that subjective visual experience is shaped by the cumulative contribution of two processes operating independently at the neural level, one reflecting visual awareness per se and the other reflecting spatial attention.

Key words: magnetoencephalography; gamma; alpha; vision; consciousness; attention

\section{Introduction}

Visual awareness, defined as the subjective sensation of seeing something (Searle, 1992; Block, 1995), is a simple form of consciousness for which a growing number of neural correlates are being proposed (Metzinger, 2000; Dehaene et al., 2001; Rees et al., 2002; Ress and Heeger, 2003; Haynes et al., 2005; Sergent et al., 2005; Schurger et al., 2006; Del Cul et al., 2007; Koivisto et al., 2007; Melloni et al., 2007). However, visual awareness remains difficult to study by itself because of its tight relationship with attention (James, 1890; Koch, 2004): faint stimuli are more easily consciously detected and identified when presented at an attended location (Posner et al., 1980), and spatial attention has even been shown to enhance the perceived contrast (Carrasco et al., 2004). What we consciously see thus seems to strongly depend on where we attend to.

However, some authors have started to point at the need to disambiguate visual awareness and spatial attention (Lamme,

Received 0ct. 19, 2007; revised Dec. 17, 2007; accepted Jan. 20, 2008

This work was supported by grants from Région lle-de-France (V.W.) and Agence Nationale de la Recherche (C.T.-B.). We thank A. Ducorps, D. Schwartz, F. Bergame, J. D. Lemarechal, and Dr. P. Pradat-Diehl for assistance with MEG recordings and data processing.

Correspondence should be addressed to Valentin Wyart, Centre National de la Recherche Scientifique, Cognitive Neuroscience and Brain Imaging Laboratory-LENA, Unité Propre de Recherche 640, 47 Boulevard de I'Hôpital, 75613 Paris cedex 13, France. E-mail: valentin.wyart@chups.jussieu.fr.

DOI:10.1523/JNEUROSCI.4748-07.2008

Copyright $\odot 2008$ Society for Neuroscience $\quad$ 0270-6474/08/282667-13\$15.00/0
2003, 2006; Koch and Tsuchiya, 2007). Recently gathered psychophysical evidence has indeed shown that the two may be dissociable under some conditions (Woodman and Luck, 2003; Kentridge et al., 2004; Sumner et al., 2006), and theoretical developments suggest distinct underlying brain mechanisms (Lamme, 2004). However, most of the proposed neural correlates of visual awareness do not explicitly distinguish top-down attention from awareness per se: consequently, experimental evidence supporting their neural dissociation has remained sparse (FernandezDuque et al., 2003; Babiloni et al., 2006; Koivisto and Revonsuo, 2007).

To address this issue, we recorded magnetoencephalographic (MEG) signals from human subjects engaged in a paradigm derived from the spatial cueing task (Posner, 1980) in which visual awareness and spatial attention were handled as distinct experimental variables (see Fig. 1A). For this purpose, we used faint stimuli that were visually constant across trials in terms of contrast and duration but were consciously seen only in half of the trials. At the beginning of each trial, subjects were cued by a predictive central arrow to covertly attend either left or right lower visual field. After $600 \mathrm{~ms}$, a faint grating appeared for 400 $\mathrm{ms}$ at either the attended or unattended location (stimuluspresent condition) or sometimes did not appear at all (stimulusabsent condition) (see Fig. $1 B$ ). Subjects had first to discriminate the orientation of the grating among two choices (orientation discrimination task) and then to report whether they believed a 
stimulus was present during the trial (detection task). We adopted classical operational definitions of visual awareness and spatial attention: the shortening of reaction times at the orientation discrimination task was considered as a behavioral measure of spatial attention, whereas subjective reports of visual experience at the detection task were considered as a behavioral measure of visual awareness. Each stimulus could be classified as attended or not (based on its location relative to the cued location) and consciously seen or not (based on the subject's report): this experimental design thus allowed for the direct factorial study of the brain events underlying visual awareness and spatial attention.

\section{Materials and Methods \\ Participants}

Twelve naive right-handed subjects with normal or corrected-to-normal vision took part in the study (six women and six men, ranging from 18 to 28 years of age). All subjects provided informed written consent and were paid for their participation. All procedures were approved by the national ethics committee (Comité Consultatif de Protection des Personnes dans la Recherche Biomédicale, Hôpital de la Pitié-Salpêtrière, Paris, France).

\section{Procedure and stimuli materials}

The stimuli used in the experiment were circular gratings (spatial frequency, 5.0 cycles per degree of visual angle; diameter, $2.0^{\circ}$ of visual angle; orientation, chosen among 20 equally spaced between 0 and $180^{\circ}$, vertical and horizontal orientations being excluded). The stimuli were positioned at $2.0^{\circ}$ of visual angle from fixation with a declination of $30^{\circ}$ in the left or right lower visual quadrants. This position has been chosen to maximize MEG responses from early visual areas (Portin et al., 1999). The Michelson contrast of the stimuli was manipulated to reach threshold levels $(<5 \%)$ using a simple software attenuator (Tyler, 1997). The left and right spatial cues only differed by three corners from the fixation cross and were subtending $0.9 \times 0.6^{\circ}$ of visual angle around fixation. The stimuli, fixation cross, and spatial cues were created off-line using Matlab (MathWorks, Natick, MA), and stimulus presentation was controlled using the Psychtoolbox package for Matlab (Brainard, 1997; Pelli, 1997). All stimuli were presented via a mirror system on a gray background (luminance, $26.9 \mathrm{~cd} / \mathrm{m}^{2}$ ) at the center of a back projection screen positioned at $85 \mathrm{~cm}$ from subjects' eyes, using a calibrated Mitsubishi X120 projector (resolution, $1024 \times 768$ pixels; refresh rate, $60 \mathrm{~Hz}$ ) located outside the shielded recording room. The luminosity of the recording room was controlled as well as the luminance of the gray background of the projection screen using a Konica Minolta LS-100 luminance meter before and after the experiment.

Each subject underwent a calibration session (mean duration, $12 \mathrm{~min}$ ) before the experiment to estimate the threshold contrast for which only half of the presented stimuli were reported as present (and thus consciously seen). The calibration session consisted of two randomly interleaved psychophysical staircases. We used one-up one-down staircases, theoretically converging toward a detection rate of $50 \%$. In practice, subjects were engaged in the same paradigm as described previously (Fig. 1 ), except that the contrast of the stimuli was varied from trial to trial depending on their previous seen-unseen report in the corresponding staircase. Threshold contrasts were thus estimated separately and simultaneously at the attended $(3.3 \pm 0.3 \%$, mean \pm SEM $)$ and unattended $(3.8 \pm 0.3 \%)$ locations. Although significant (Wilcoxon's signed-rank test, $p=0.04$ ), the modulation of threshold contrast by spatial attention was quite small. Therefore, we used the estimate of threshold contrast at the attended location for all stimuli presented to the subject during the recording sessions.

The calibration session was followed by eight recording sessions (mean

\section{Experimental design}

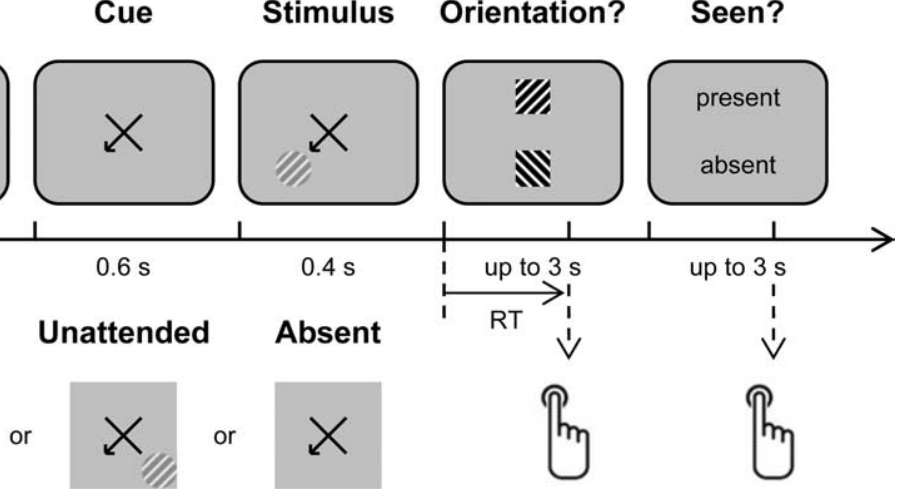
Figure 1. Experimental design. $A$, Paradigm. At the beginning of each trial, subjects were cued by a predictive central arrow to
covertly attend either left or right lower visual field. After $600 \mathrm{~ms}$, a faint grating appeared for $400 \mathrm{~ms}$ either at the attended or unattended location or sometimes did not appear at all. Subjects had first to discriminate the orientation of the grating among two choices and then to report whether they believed a stimulus was present during the trial. B, Stimuli. Eighty-five percent of the s contained a stimulus (stimulus-present condition) that could appear either at the attended or unattended location (cue

duration, 8 min per session). Each of the eight recording sessions consisted of 92 trials, including 80 stimulus-present trials (in which stimuli at threshold contrast were presented either in the left or right lower visual quadrants) and 12 stimulus-absent trials (in which no stimulus was presented). Among the stimulus-present trials, 52 of the 80 stimuli were presented at the cued location (attended condition, cue validity, 65\%), whereas 28 were presented at the uncued location (unattended condition). Trials within a recording session were presented in a different randomized order for each subject.

After stimulus presentation, subjects were first asked to discriminate as quickly and accurately as possible the orientation of the grating among two possibilities distant by $60^{\circ}$. The two choices were presented vertically: if the trial contained a stimulus, then one of the two orientations corresponded to the orientation of the presented grating; otherwise, the two orientations were chosen randomly (but spaced by $60^{\circ}$ ). Subjects pressed the upper response button with their index finger to choose the upper orientation or the lower response button with their middle finger to choose the lower orientation. After a manual response, or after $3 \mathrm{~s}$ without response ( $<1 \%$ of all trials), subjects were asked to report whether or not they believed a stimulus was presented during the trial. The words "present" and "absent" were presented vertically, their respective positions being randomized across trials. Subjects were asked to choose "present" when they believed that a stimulus had been presented during the trial, whether they could discriminate its orientation or not (consciously seen condition), and choose "absent" otherwise (unseen condition). Subjects pressed the upper response button with their index finger to choose the upper report or the lower response button with their middle finger to choose the lower report. After a variable delay of 2-3 s, the next trial began.

We delayed introspective reports of subjects' visual experience after factual reports about the orientation of the stimulus to urge subjects to choose (or guess) one of the two orientations irrespectively of whether they had low confidence in their choice (Block, 2005). Subjects were specifically asked to perform the orientation discrimination task in all trials. When they did not believe a stimulus was presented during the trial (i.e., half of the time), they were asked to give their first guess. Although some subjects reported feeling uneasy guessing during the calibration session, this frequent situation became "natural" before the recording sessions began. Importantly, subjects responded to the orientation discrimination task as often when the stimuli were consciously seen as when they were not (Wilcoxon's signed-rank test, $p>0.2$, NS). We used a variable stimulus-response mapping scheme (e.g., "present" reports randomly appeared at either the upper or the lower position on the screen) that prevented subjects from learning fixed sensorimotor associations 
(Dobbins et al., 2004) and provided a clear temporal separation between neural processes related to stimulus processing and those related to the execution of the motor response. During stimulus presentation, subjects thus gathered as much sensory information about the stimulus as possible without knowing which of the two response buttons they would have to press afterward.

Feedback regarding subjects' performance at the orientation discrimination task (correct discrimination rate and mean reaction time) was provided at the end of each session. Subjects were not given any explicit feedback regarding either the proportion of stimulus-absent trials or the presence of a stimulus at the end of each trial, because such information could have introduced undesirable decision biases in the subsequent reports of awareness.

\section{MEG recordings}

Continuous magnetoencephalographic signals were collected using a whole-head MEG system with 151 axial gradiometers (CTF Systems, Port Coquitlam, British Columbia, Canada) at a sampling rate of $1250 \mathrm{~Hz}$ and low-pass filtered on-line at $300 \mathrm{~Hz}$. Head localization was tracked during the experiment with respect to the MEG sensors using marker coils that were placed at the cardinal points of the head (nasion, left and right ear). Vertical and horizontal electrooculogram (EOG) signals were simultaneously collected.

\section{Data analysis}

Data preprocessing, analysis, and visualization were performed using both in-house software (http://cogimage.dsi.cnrs.fr/logiciels/) and additional programs developed in Matlab (MathWorks). All time samples were corrected with respect to the refresh delay of the projector $(+25 \mathrm{~ms}$, measured on-line with a photodiode).

Artifact rejection. Trials contaminated with eyes movements (rejection threshold, $1.0^{\circ}$ of visual angle from fixation), eye blinks, or muscular artifacts were rejected off-line on visual inspection of their unfiltered EOG and MEG traces. We used a simple voluntary saccade task toward the locations of the stimuli to calibrate the EOG for each subject: the chosen rejection threshold for eye movements corresponded to a deflection of $\pm 20 \mu \mathrm{V}$ in $<30 \mathrm{~ms}$ on the horizontal EOG traces. Eye blinks are easily detectable on the vertical EOG traces and could be removed automatically (rejection threshold, $90 \mu \mathrm{V}$ ). In contrast, muscular artifacts produce strong high-frequency artifacts on temporo-occipital MEG traces that are not easily detected by automatic procedures. Because we focused our analysis on gamma-band (i.e., high-frequency) activity, we took great care to remove all trials containing such artifacts. Besides, two malfunctioning MEG sensors $\left(\mathrm{MRF}_{21}\right.$ overlying right frontal cortex and $\mathrm{MRT}_{32}$ overlying right temporal cortex) were discarded from all analyses.

Time-frequency analysis. A time-frequency wavelet transform was applied to each trial at each MEG sensor using a family of complex Morlet wavelets $(m=10)$, resulting in an estimate of signal power at each time sample and at each frequency between 8 and $150 \mathrm{~Hz}$, ranging from alpha $(8-14 \mathrm{~Hz}$; frequency step, $0.2 \mathrm{~Hz})$ to gamma $(30-150 \mathrm{~Hz}$; frequency step, $2 \mathrm{~Hz}$ ) frequency bands. Importantly, the time-frequency resolution of the wavelets was frequency dependent $(10 \mathrm{~Hz}: \sigma=150 \mathrm{~ms}, 1 \mathrm{~Hz}$; $50 \mathrm{~Hz}$ : $\sigma=30 \mathrm{~ms}, 5 \mathrm{~Hz}$, with FWHM $=2.355 \sigma$ the full-width at half-maximum of the Gaussian envelope of the wavelet). The time-frequency transformed data were then averaged across trials for each of the conditions and for each subject. Thus, estimates of signal power contained components both phase locked (i.e., evoked) and non-phase locked (i.e., induced) to stimulus onset (Tallon-Baudry and Bertrand, 1999). The timefrequency data during the last $200 \mathrm{~ms}$ of the fixation period preceding each trial ( 200 to $0 \mathrm{~ms}$ precue, i.e., 800 to $600 \mathrm{~ms}$ prestimulus) was used as baseline. An index of signal power, defined at each time sample and frequency as the base-2 log-transformed increase of signal power relative to baseline, was considered as the measure of interest for all time-frequency analyses. Indeed, the log-transformed data were distributed normally, which allowed us to use standard parametric tests (e.g., paired $t$ tests, repeated-measures ANOVAs) to assess the statistical significance of the observed effects (Schurger et al., 2006).

Splitting of the time-frequency data. We split the time-frequency data into eight conditions along three experimental factors: stimulus (left or right position), visual awareness (consciously seen or unseen condition), and spatial attention (attended or unattended condition). Importantly, we did not consider the accuracy of subjects' responses at the orientation discrimination task as an additional experimental factor: instead, we considered as consciously seen the stimuli that were both reported as present and correctly identified and as unseen all stimuli that were reported as absent. This splitting of the time-frequency data gave approximately equal sample sizes for consciously seen and unseen conditions while keeping most of the recorded trials. However, to confirm that awareness-related effects did not actually correspond to accuracy-related effects, we further tested whether these effects could be attributable to the bias in discrimination accuracy between consciously seen and unseen conditions, as described in Results.

Statistical analysis. We first analyzed the time-frequency data using standard parametric tests (e.g., paired $t$ tests, repeated-measures ANOVAs) performed at many MEG sensors or many data points in the timefrequency domain. In this situation of multiple comparisons, we further assessed the statistical significance of observed effects using ad hoc nonparametric randomization tests (Nichols and Holmes, 2002; Jokisch and Jensen, 2007; Medendorp et al., 2007). This family of statistical tests allowed us to control the type I error rate with respect to multiple comparisons by evaluating the original effect at the group level under its randomized null distribution, in other words, by estimating the probability that the original effect was obtained by chance. The nonparametric randomization tests used for the identification and the factorial analysis of the gamma-band response to the faint stimuli are fully described below.

Each of these tests corresponded to a random-effects analysis, because the randomization procedure was performed at the level of subjects rather than at the level of single trials. All nonparametric randomization tests also provided an implicit correction with respect to unequal sample sizes between conditions (e.g., between attended and unattended conditions), because each subject's data were randomly allocated to one of the possible conditions at the single-trial level while keeping the same (that is, potentially unequal) sample sizes as originally obtained for the subject (Schurger et al., 2006). The resulting two-tailed $p$ values were thus corrected with respect both to multiple comparisons and to unequal sample sizes between conditions and are subsequently referred to as $p_{c}$.

Statistical analysis identification of the gamma-band response. We identified the group of MEG sensors showing a significant increase of gamma-band activity during stimulus presentation relative to baseline. First, we selected the group of contiguous sensors showing a significant increase of gamma-band activity in the time-frequency window of interest using paired $t$ tests against zero mean performed separately for every sensor with a threshold at $p<0.01$. Then, we assessed the statistical significance of this increase at the group level (i.e., across the contiguous sensors) using a nonparametric randomization test (Jokisch and Jensen, 2007). For this purpose, we defined a group-level test statistic $\Sigma_{T}$ as the sum of the $T$ scores of all selected sensors, and we controlled the corresponding type I error rate by evaluating the original $\Sigma_{T}$ under its randomized null distribution. The null distribution was estimated by repeating the following randomization procedure many times $(n=10,000)$ : (1) permuting each subject's baseline and active time windows at the singletrial level; and (2) computing the corresponding randomized $\Sigma_{T}$. Based on the estimated null distribution, we computed the two-tailed $p$ value $p_{\mathrm{c}}$ of a randomized $\Sigma_{T}$ exceeding the original $\Sigma_{T}$.

Statistical analysis: factorial analysis of the gamma-band response. We computed the time-frequency representations of the separate main effects of visual awareness (consciously seen - unseen stimulus) and spatial attention (attended - unattended stimulus) on the gamma-band response. As a preprocessing step, we applied a sliding time-frequency average to the gamma-band response, ranging from 0 to $500 \mathrm{~ms}$ after stimulus and from 40 to $120 \mathrm{~Hz}$ (window, $100 \mathrm{~ms}, 2 \mathrm{~Hz}$; step, $5 \mathrm{~ms}, 2 \mathrm{~Hz}$ ). We computed the $F$ scores of the main effects of visual awareness and spatial attention using repeated-measures ANOVAs performed separately for every data point in the time-frequency domain. Then, we assessed the statistical significance of the selected awareness- and attentionrelated effects at the group level (i.e., across the neighboring data points in the time-frequency domain) using a nonparametric randomization 
test (Medendorp et al., 2007). For this purpose, we defined a group-level test statistic $\Sigma_{F}$ as the sum of the $F$ scores of all data points in the selected time-frequency window of interest, and we controlled the corresponding type I error rate by evaluating the original $\Sigma_{F}$ under its randomized null distribution. The null distribution was estimated by repeating the following randomization procedure many times $(n=$ 10,000): (1) randomly allocating each subject's data to one of the four conditions at the singletrial level while keeping the same sample sizes as originally obtained for the subject; and (2) computing the corresponding randomized $\Sigma_{F}$. Based on the estimated null distribution, we computed the two-tailed $p$ value $p_{c}$ of a randomized $\Sigma_{F}$ exceeding the original $\Sigma_{F}$.

\section{Results}

Subjective reports define two distinct cognitive states

Facing visually constant stimuli, subjects were found to fluctuate randomly between two distinct cognitive states: an aware state in which subjects reported seeing the stimulus and could reliably identify its orientation (corresponding to the consciously seen condition) and an unaware state in which subjects did not see the stimulus and could not even guess its orientation better than chance (corresponding to the unseen condition). First of all, subjects did not answer randomly: they reported the presence of a stimulus much more often when the stimulus was actually present (detection rate, $46.6 \pm 2.5 \%$, mean \pm SEM) than absent (false-alarm rate, $14.9 \pm 2.6 \%$ ). This corresponds to a significantly positive detection sensitivity index $d^{\prime}{ }_{\text {det }}=1.04 \pm 0.14\left(t\right.$ test against zero mean, $T_{(11)}=7.3, p<$ 0.001 ) along with a conservative (i.e., positive) decision criterion $\log \beta=+0.69 \pm 0.14$ ( $t$ test against zero mean, $T_{(11)}=4.9, p<$ 0.001 ), according to signal detection theory (Green and Swets, 1966; Macmillan and Creelman, 2005).

Moreover, subjects performed significantly better at the orientation discrimination task when they reported seeing the stimulus (paired $t$ test, $T_{(11)}=9.9, p<0.001$ ). Indeed, $84.5 \pm 3.2 \%$ of consciously seen stimuli were correctly identified (i.e., a discrimination sensitivity index $d^{\prime}{ }_{\text {dis }}=1.54$ ), which was significantly better than chance (Kolmogorov-Smirnov test, binomial, $p<0.001$ ). In contrast, only $51.7 \pm 0.9 \%$ of unseen stimuli were correctly identified (i.e., $d^{\prime}{ }_{\text {dis }}=0.17$ ), which was not significantly better than chance (Kolmogorov-Smirnov test, binomial, $p=0.18$, NS). Such a match between subjective (reports of visual experience at the detection task) and objective (accuracy at the orientation discrimination task) thresholds of conscious perception suggest that aware and unaware states do not merely reflect a response bias but rather characterize intrinsically different cognitive states (Hannula et al., 2005). Furthermore, the temporal sequence of seen-unseen reports did not show any bias of probability toward one of the two possible choices across consecutive trials (Wilcoxon's signed-rank test, $P\left[\operatorname{report}_{(N)}=\right.$ $\left.\operatorname{report}_{(N-1)}\right]$ minus $P\left[\operatorname{report}_{(N)} \neq \operatorname{report}_{(N-1)}\right], p>0.5$, NS), which further argues against a simple response bias between seen and unseen reports.

\section{A complex interaction scheme between visual awareness and spatial attention}

Subjects' behavior revealed a complex interaction scheme between visual awareness and spatial attention. On the one hand, spatial attention improved subjects' ability to consciously perceive the stimuli, because $49.9 \pm 3.1 \%$ were consciously seen at the attended location against $40.4 \pm 3.0 \%$ at the unattended location (paired $t$ test, $T_{(11)}=2.5, p=0.03$ ). On the other hand, visual awareness appeared necessary for spatial attention to shorten reaction times. Indeed, reaction times at the orientation discrimination task were significantly shorter when stimuli were presented at the attended location (repeated-measures ANOVA; main effect: attention, $\left.F_{(1,11)}=6.2, p=0.03\right)$. However, the amplitude of this attentional cueing effect critically depended on stimulus awareness (attention $\times$ awareness interaction, $F_{(1,11)}=$ $6.4, p=0.03$ ). Spatial attention significantly shortened reaction times to consciously seen stimuli (post hoc comparison, attended - unattended stimulus $=-47.1 \pm 13.0 \mathrm{~ms}, F_{(1,11)}=$ $13.0, p=0.004)$ but did not affect reaction times to unseen stimuli ( post hoc comparison, attended - unattended stimulus $=$ $-10.5 \pm 14.2 \mathrm{~ms}, F_{(1,11)}=0.5, p>0.5$, NS). Visual awareness and spatial attention thus appeared mutually dependent at the behavioral level. But does this interaction scheme also hold true at the neural level, particularly during the visual processing of the faint stimuli?

\section{Visual awareness does not reflect the orienting of spatial attention}

We first assessed whether the lack of conscious perception in half of the stimulus-present trials could be attributable to a failure of the orienting of spatial attention in those trials. Because modulations of posterior alpha-band oscillatory activity $(8-14 \mathrm{~Hz})$ have been shown previously to index spatial biases of attention (Worden et al., 2000; Sauseng et al., 2005; Thut et al., 2006; Medendorp et al., 2007), we measured alpha-band activity at the time when subjects oriented their attention in response to the central cue, before stimulus onset. The average power frequency spectrum of all posterior MEG sensors $(n=$ 42 sensors overlying occipital, parietal, and temporal cortices) showed a peak at $11 \mathrm{~Hz}$. Consequently, we defined a frequency band of interest in the alpha frequency range at 10-12 Hz. Subjects showed a strong decrease of alpha-band activity over bilateral posterior sensors at 300-600 ms after cue, which was more pronounced contralaterally to the cued location (Fig. 


\section{Stimulus-related $\mathrm{Y}$-band activity}

(50-110 Hz, 150-500 ms post-stimulus)

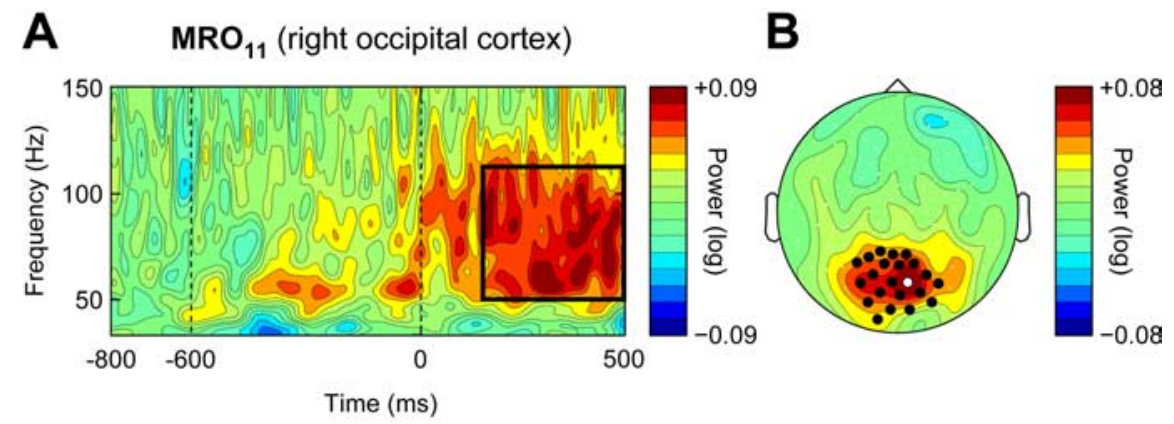

\section{Awareness-related effect}
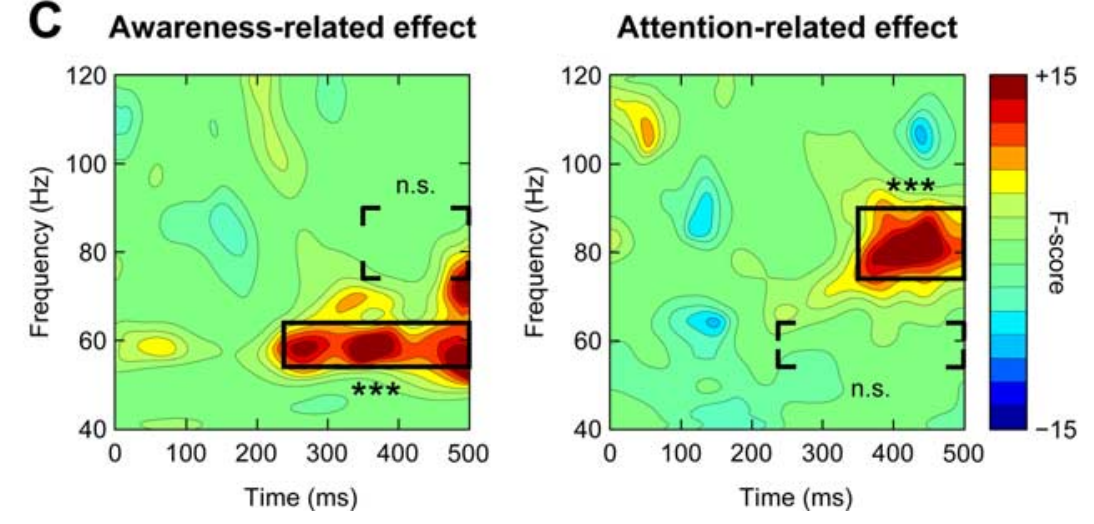

Figure 3. Stimulus-related gamma-band activity. $\boldsymbol{A}$, Time-frequency representation of the gamma-band response of the $M E G$ sensor showing the strongest gamma-band activity: $\mathrm{MRO}_{11}$, overlying right occipital cortex. The time-frequency window of interest was defined at $50-110 \mathrm{~Hz}$ and $150-500 \mathrm{~ms}$ after stimulus (black box). B, Scalp topography of the gamma-band response. Thick sensors indicate the responding ROI $(n=22)$, corresponding to the group of sensors showing the strongest increase of gamma-band activity in response to the faint stimuli (white sensor, $\mathrm{MRO}_{11}$ ). C, Factorial analysis of the gamma-band response in the time-frequency domain. Left, Awareness-related effect. A mid-frequency component of the gamma-band response $(54-64 \mathrm{~Hz})$ showed a main effect of visual awareness independent of spatial attention (awareness-related activity). Right, Attention-related effect. A high-frequency component of the gamma-band response $(76-90 \mathrm{~Hz})$ showed a main effect of spatial attention independent from visual awareness (attention-related activity). F scores were signed by the direction of the corresponding main effect. ${ }^{* * *} p_{c}<0.001$ (black boxes); n.s., a nonsignificant effect (dashed boxes).

\section{Identification of the gamma-band response to the faint stimuli}

We then aimed at identifying a reliable neural response to the faint stimuli used. We focused our analysis on gamma-band oscillatory activity $(30-150 \mathrm{~Hz})$, a neural correlate of recurrent synchronization at the population level observed at several recording scales (Singer and Gray, 1995; Tallon-Baudry and Bertrand, 1999; Fries et al., 2007), because this mechanism has been functionally related both to visual awareness (Engel and Singer, 2001; Fries et al., 2002; Schurger et al., 2006; Wilke et al., 2006) and spatial attention (Gruber et al., 1999; Fries et al., 2001; Landau et al., 2007; Womelsdorf and Fries, 2007). As a preprocessing step, we averaged gamma-band activity across all subjects and conditions containing a stimulus. This grandaveraging process provided us with the unbiased mean gamma-band response to the faint stimuli, independently of visual awareness and spatial attention.

First, we identified the MEG sensor showing the strongest increase of gammaband activity during stimulus presentation (30-150 Hz, 0-500 ms after stimulus) relative to baseline ( $800-600 \mathrm{~ms}$ before stimulus): MEG right occipital sensor 11 $\left(\mathrm{MRO}_{11}\right)$, overlying right occipital cortex. Based on the time-frequency representation of the gamma-band response at this sensor, we defined a broad time-frequency window of interest in the gamma frequency range at $50-110 \mathrm{~Hz}$ and $150-500 \mathrm{~ms}$ after stimulus (Fig. 3A). Then, we extracted the group of $n=22$ contiguous parietooccipital sensors showing a significant gamma-band response in the time-frequency window of interest $(50-110 \mathrm{~Hz}$,

$2 A$ ). We thus defined two groups of $n=11$ contiguous posterior sensors contralateral to the cued location showing the strongest orienting-related effect at $300-600 \mathrm{~ms}$ after cue (paired $t$ test, left cue - right cue) (Fig. 2B). We will further refer to this group of sensors as the orienting-related region of interest (ROI). We then performed the repeated-measures ANOVA of this activity along the experimental factors cue (left or right cue) and hemisphere (ipsilateral or contralateral to cue). This statistical test confirmed the statistical significance of the contralateral lateralization effect (main effect: hemisphere, $\left.F_{(1,11)}=48.9, p<0.001\right)$.

However, the amplitude of this contralateral decrease of posterior alpha-band activity did not significantly differ whether subjects would consciously see upcoming stimuli presented at the cued location (post hoc comparison, contralateral to valid cue, consciously seen - unseen stimulus, $F_{(1,11)}=0.1, p>0.5$, NS). This result suggests that unseen stimuli did not go unnoticed simply because subjects failed to orient their attention toward the cued location. Therefore, subjects consistently paid attention to the cued location, and visual awareness does not appear to merely reflect the orienting of spatial attention toward the location of the upcoming stimulus.
$150-500 \mathrm{~ms}$ after stimulus) using a nonparametric randomization test $\left(p_{\mathrm{c}}<0.001\right.$ corrected for multiple comparisons across contiguous sensors; see Materials and Methods) (Fig. 3B). We will further refer to this group of sensors as the responding ROI. Varying the boundaries of the time-frequency window of interest $( \pm 10 \mathrm{~Hz}, \pm 50 \mathrm{~ms})$ did not change the topography of the responding ROI. Therefore, the faint stimuli used here induced a weak yet reliable gamma-band response over the visual cortex. Similar parieto-occipital increases of gamma-band activity have been observed previously in the scalp MEG using visual stimuli at maximal contrast (Hoogenboom et al., 2006; Vidal et al., 2006; Siegel et al., 2007).

\section{Factorial analysis of the gamma-band response}

To determine whether this neural response was functionally related to visual awareness and spatial attention, we conducted its factorial analysis in the time-frequency domain. Precisely, we computed the time-frequency representations of the separate main effects of visual awareness (consciously seen - unseen stimulus) and spatial attention (attended - unattended stimulus) on the gamma-band response $(50-110 \mathrm{~Hz}, 150-500 \mathrm{~ms}$ after stimulus) over the responding ROI using multiple repeated- 
measures ANOVAs. We assessed the statistical significance of the observed awareness- and attention-related effects using a nonparametric randomization test (correcting for multiple comparisons across neighboring data points in the timefrequency domain; see Materials and Methods).

This factorial analysis of the gammaband response revealed a neural dissociation between visual awareness and spatial attention at different latencies and frequencies (Fig. 3C). Indeed, a midfrequency component $(54-64 \mathrm{~Hz})$ of the gamma-band response showed a significant main effect of visual awareness independent of spatial attention at $240-500 \mathrm{~ms}$ after stimulus (main effect: awareness, $p_{\mathrm{c}}<$ 0.001; main effect: attention, $p>0.5$, NS; interaction: awareness $\times$ attention, $p>$ 0.5 , NS). Conversely, a high-frequency component $(76-90 \mathrm{~Hz})$ of the gammaband response showed a significant main effect of spatial attention independent of visual awareness at 350-500 ms after stimulus (main effect: attention, $p_{\mathrm{c}}<0.001$; main effect: awareness, $p>0.2$, NS; interaction: awareness $\times$ attention, $p=0.18$, NS). We will further refer to these midfrequency and high-frequency components of the gamma-band response as the awareness- and attention-related gammaband activities, respectively.

Awareness-related gamma-band activity The awareness-related gamma-band activity (54-64 Hz, 240-500 ms after stimulus) peaked over contralateral occipital MEG sensors in response to consciously seen stimuli (Fig. 4A). Within the responding ROI, we thus defined two nonoverlapping groups of $n=8$ contiguous occipital sensors contralateral to the location of the stimulus showing the strongest midfrequency gamma-band response to consciously seen stimuli, further referred to as the awareness-related ROI. We then performed the repeated-measures ANOVA of the awareness-related gamma-band activity $(54-64 \mathrm{~Hz}, 240-500 \mathrm{~ms}$ after stimulus, awareness-related ROI) along the following experimental factors: stimulus (left or right location), hemisphere (ipsilateral or contralateral to stimulus), visual awareness (consciously seen or unseen stimulus), and spatial attention (attended or unattended stimulus).

This statistical analysis confirmed the contralateral lateralization of this activity (post hoc comparison, ipsilateral - contralateral to consciously seen stimulus, $\left.F_{(1,11)}=25.5, p<0.001\right)$ but also showed that the level of the contralateral activity diverged strongly between consciously seen and unseen stimuli (post hoc comparison, contralateral to stimulus, consciously seen - unseen stimulus, $\left.F_{(1,11)}=31.0, p<0.001\right)$. Consciously seen stimuli induced a strong mid-frequency oscillatory response whereas un-

\section{Awareness-related Y-band activity}

\author{
(54-64 Hz, 240-500 ms post-stimulus)
}

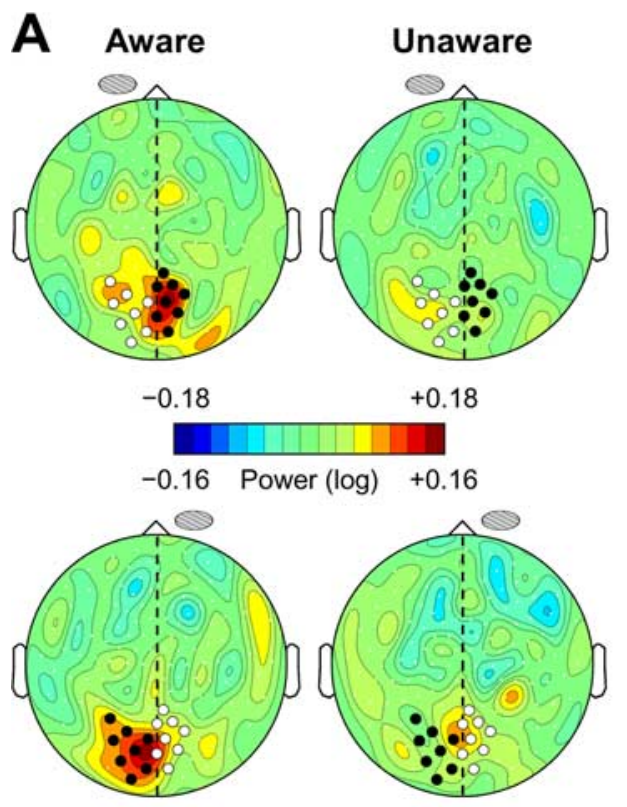

B

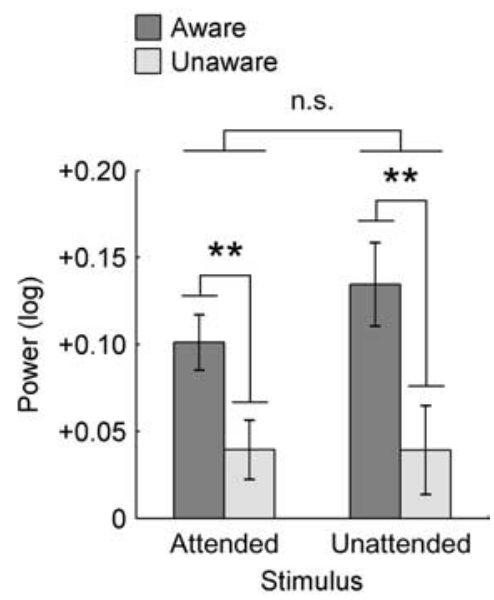

Attention-related effect
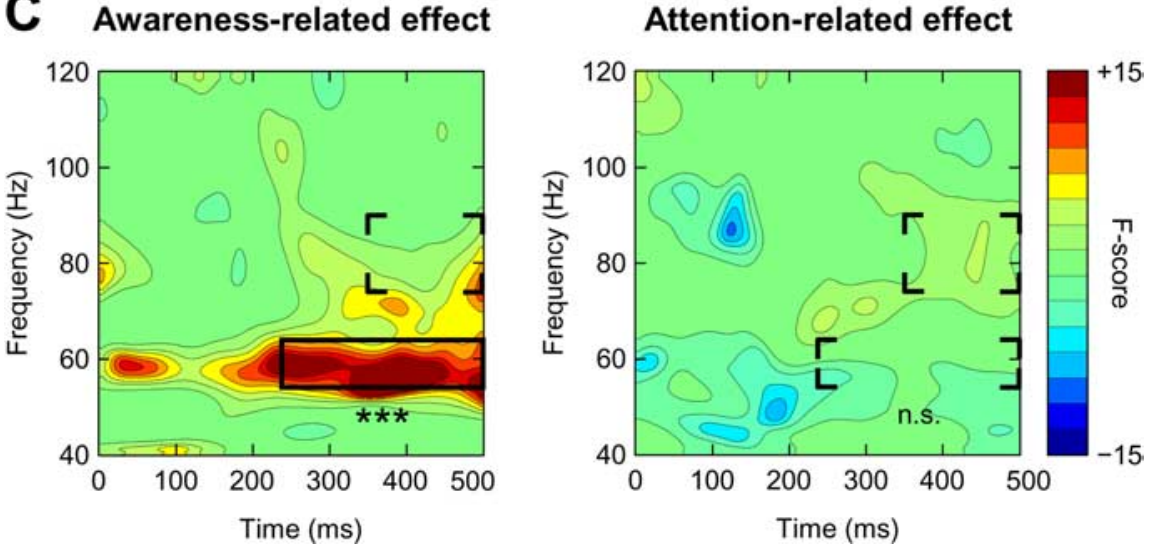

Figure 4. Awareness-related gamma-band activity. $\boldsymbol{A}$, The awareness-related gamma-band activity $(54-64 \mathrm{~Hz}, 240-500$ ms after stimulus) peaked contralaterally to the stimuli (first row, left stimulus; second row, right stimulus) and was strongly increased in response to consciously seen stimuli. Thick sensors indicate the awareness-related ROI, corresponding to the peak of this activity ( $n=8$ for each hemisphere; white, ipsilateral to stimulus; black, contralateral to stimulus). $\boldsymbol{B}$, Average $54-64 \mathrm{~Hz}$ power over the awareness-related ROl at $240-500 \mathrm{~ms}$ after stimulus. The awareness-related gamma-band activity was strongly increased for consciously seen stimuli and was not modulated by spatial attention. C, Factorial analysis of the gamma-band response over the awareness-related ROI. The main effect of visual awareness was present only in the mid-frequency band. № clear effect of spatial attention was found over this region, whether in the mid-frequency or high-frequency bands. Error bars indicate SEM. ${ }^{* *} p<0.01 ;{ }^{* * *} p<0.001 ;$ n.S., a nonsignificant effect.

seen stimuli did not, which corresponded to a 200\% (approximately threefold) increase of baseline-corrected power in the consciously seen condition compared with the unseen condition (Fig. $4 B$ ). Importantly, the amplitude of this awareness-related effect did not differ whether the stimuli were presented inside or outside the focus of spatial attention ( post hoc comparison, contralateral to stimulus, interaction: awareness $\times$ attention, $F_{(1,11)}$ $=0.0, p>0.5, \mathrm{NS})$. The overall power of the contralateral activity was not significantly modulated by spatial attention in this frequency band, even when considering consciously seen stimuli only (post hoc comparison, contralateral to consciously seen stimulus, attended - unattended stimulus, $F_{(1,11)}=1.8, p>0.2$, NS). When constrained to the awareness-related ROI, the facto- 


\section{Attention-related $\mathbf{y - b a n d}$ activity}

\author{
(76-90 Hz, 350-500 ms post-stimulus)
}

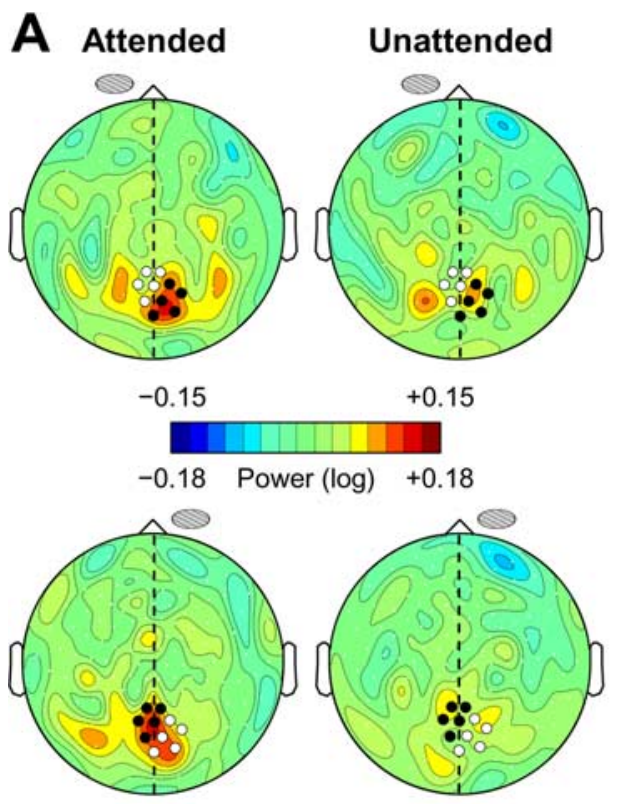

B

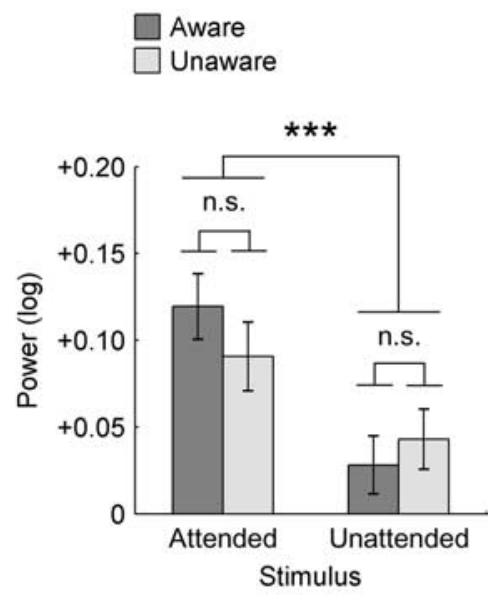

C Awareness-related effect
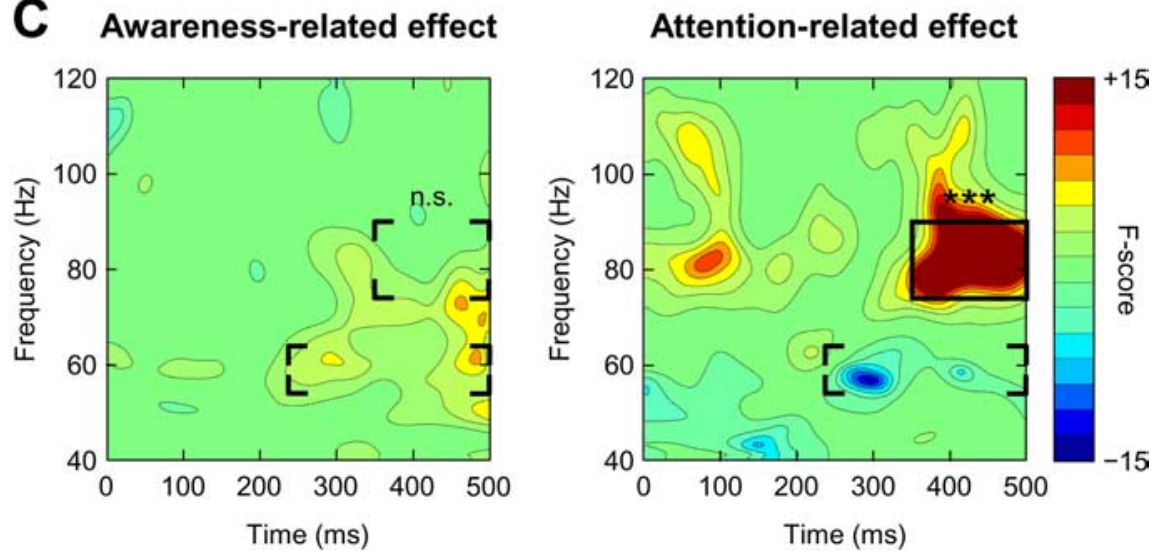

Figure 5. Attention-related gamma-band activity. $\boldsymbol{A}$, The attention-related gamma-band activity $(76-90 \mathrm{~Hz}, 350-500 \mathrm{~ms}$ after stimulus) peaked contralaterally to the stimuli (first row, left stimulus; second row, right stimulus) and was strongly increased when stimuli were presented at the attended location. Thick sensors indicate the attention-related Rol, corresponding to the peak of this activity ( $n=5$ for each hemisphere; white, ipsilateral to stimulus; black, contralateral to stimulus). $\boldsymbol{B}$, Average $76-90 \mathrm{~Hz}$ power over the attention-related ROI at 350-500 ms after stimulus. Attended stimuli induced a larger high-frequency gamma-band activity than unattended stimuli, whether those stimuli were consciously seen or not. $\boldsymbol{C}$, Factorial analysis of the gamma-band response over the attention-related ROl. The main effect of spatial attention was present only in the highfrequency band. No clear effect of visual awareness was found over this region, whether in the mid-frequency or high-frequency bands. Error bars indicate SEM. ${ }^{* *} p<0.001$; n.S., a nonsignificant effect.

rial analysis of the gamma-band response in the time-frequency domain confirmed the main effect of visual awareness in the mid-frequency band only but showed no main effect of spatial attention, whether in the mid-frequency or high-frequency bands (Fig. 4C).

Although independent of spatial attention, we further assessed whether this awareness-related effect on the gamma-band response could also be attributable to the much higher discrimination accuracy in the consciously seen condition. For this purpose, we first split the unseen condition into correct and incorrect responses and tested these two datasets against each other. No significant difference was found (repeated-measures ANOVA, main effect: accuracy, $F_{(1,11)}=0.8, p>0.2$, NS), which suggests that the awareness-related effect did not correspond to an accuracy-related effect. To confirm this conclusion, we then conducted an additional comparison between consciously seen and unseen trials but only for correct responses. Comparing correct trials only did not affect the strength of the awareness-related effect (repeatedmeasures ANOVA, main effect: awareness, $\left.F_{(1,11)}=19.2, p=0.001\right)$. However, because the consciously seen condition contained significantly more correct trials than the unseen condition, we checked whether the unequal sample sizes could have affected the previous comparison by repeating the following permutation procedure many times $(n=10,000)$ : ( 1$)$ for each subject, randomly extracting as many correct trials in the consciously seen condition as there were in the unseen condition; and (2) comparing this subset of correct trials in the consciously seen condition with all correct trials in the unseen condition using a repeated-measures ANOVA. The median of the obtained $p$ value distribution was significant $\left(F_{(1,11)}=19.1, p=0.001\right)$ and only 7 of $10,000 p$ values were higher than 0.05 . Therefore, the awareness-related effect on the mid-frequency gamma-band response did not depend on subjects' discrimination accuracy, as observed in blindsight patient GY (Schurger et al., 2006).

Attention-related gamma-band activity The attention-related gamma-band activity $(76-90 \mathrm{~Hz}, 350-500 \mathrm{~ms}$ after stimulus) peaked over contralateral parieto-occipital MEG sensors in response to attended stimuli (Fig. 5A). Within the responding ROI, we thus defined two nonoverlapping groups of $n=5$ contiguous parietooccipital sensors contralateral to the location of the stimulus showing the strongest attention-related gamma-band activity in response to attended stimuli, further referred to as the attention-related ROI. The awareness- and attention-related ROIs were partially overlapping but nevertheless distinct. We then performed the repeatedmeasures ANOVA of the attention-related gamma-band activity $(76-90 \mathrm{~Hz}, 350-500$ ms after stimulus, awareness-related ROI) along the same experimental factors as for the awareness-related activity: stimulus, hemisphere, visual awareness, and spatial attention.

Like the awareness-related activity, this activity was significantly lateralized contralaterally to the location of the stimulus (post hoc comparison, ipsilateral - contralateral to attended stimulus, $\left.F_{(1,11)}=10.3, p=0.008\right)$, but the level of the contralateral activity was selectively and significantly increased by spatial attention ( post hoc comparison, contralateral to stimulus, attended - unattended stimulus, $\left.F_{(1,11)}=94.2, p<0.001\right)$. Stimuli presented at the attended location induced a strong highfrequency oscillatory response, whereas stimuli presented at the 
unattended location did not, which corresponded to a $180 \%$ (approximately threefold) increase of baseline-corrected power in the attended condition compared with the unattended condition (Fig. 5B). Importantly, this effect of spatial attention did not significantly differ in amplitude whether stimuli were consciously seen or not ( post hoc comparison, contralateral to stimulus, interaction: awareness $\times$ attention, $F_{(1,11)}=1.8, p>0.2$, NS): spatial attention thus significantly increased the highfrequency gamma-band response to unseen stimuli (post hoc comparison, contralateral to unseen stimulus, attended - unattended stimulus, $\left.F_{(1,11)}=4.9, p=0.04\right)$. When constrained to the attention-related ROI, the factorial analysis of the gamma-band response in the time-frequency domain confirmed the main effect of spatial attention in the high-frequency band only but showed no main effect of visual awareness, whether in the midfrequency or high-frequency bands (Fig. 5C).

\section{Time courses of the awareness- and attention-related activities}

Because the latencies of the awareness- and attention-related effects on the gamma-band response appeared to be different (Fig. $3 C$ ), we further analyzed the respective time courses of the midfrequency and high-frequency gamma-band activities (54-64 and $76-90 \mathrm{~Hz}$, respectively). For this purpose, we defined an early (250-350 ms after stimulus) and a late (400-500 ms after stimulus) time windows. We then performed the repeatedmeasures ANOVAs of the mid-frequency and high-frequency gamma-band activities over their respective ROIs (awarenessand attention-related ROI, respectively) along the additional experimental factor time (early or late time window).

This statistical analysis confirmed that both the midfrequency and high-frequency gamma-band activities were lateralized contralaterally to the location of the stimulus but further showed that this was the case both in the early and late time windows ( post hoc comparison, ipsilateral - contralateral to stimulus, early time window, mid-frequency activity: $F_{(1,11)}=$ 11.3, $p=0.006$; high-frequency activity: $F_{(1,11)}=7.9, p=0.01$; late time window, mid-frequency activity: $F_{(1,11)}=8.1, p=0.01$; high-frequency activity: $\left.F_{(1,11)}=7.5, p=0.01\right)$ and that this lateralization did not change over time (interaction: hemisphere $\times$ time, mid-frequency activity: $F_{(1,11)}<0.1, p>0.5$, NS; high-frequency activity: $F_{(1,11)}=0.7, p>0.2$, NS). This result suggests that the mid-frequency and high-frequency gammaband activities reflected sustained stimulus processing in retinotopic visual areas at $250-500 \mathrm{~ms}$ after stimulus.

However, the influences of visual awareness and spatial attention on the mid-frequency and high-frequency gamma-band activities differed along the time dimension. The awareness-related effect on the mid-frequency gamma-band activity was present both in the early and late time windows (post hoc comparison, consciously seen - unseen stimulus, early time window: $F_{(1,11)}=$ $8.9, p=0.01$; late time window: $\left.F_{(1,11)}=14.7, p=0.002\right)$ and did not change over time (interaction: awareness $\times$ time, $F_{(1,11)}=$ $0.2, p>0.5$, NS). In contrast, the attention-related effect on the high-frequency gamma-band response was present only in the late time window ( post hoc comparison, attended - unattended stimulus, early time window: $F_{(1,11)}=2.5, p=0.14$, NS; late time window: $\left.F_{(1,11)}=44.4, p<0.001\right)$, and the amplitude of this effect increased significantly over time (interaction: attention $X$ time, $\left.F_{(1,11)}=7.8, p=0.01\right)$. Therefore, the sustained awarenessand attention-related gamma-band activities had different time courses during the visual processing of the faint stimuli.

To summarize, contrasting experimental factors allowed us to identify distinct neural correlates of visual awareness and spatial attention in time, space, and frequency. First, the posterior decrease in alpha-band activity $(10-12 \mathrm{~Hz}, 300-600 \mathrm{~ms}$ after cue, orienting-related ROI) indexed the orienting of spatial attention and did not differ whether upcoming stimuli were consciously seen or not. Second, a mid-frequency component of the gammaband response to the faint stimuli $(54-64 \mathrm{~Hz}, 240-500 \mathrm{~ms}$ after stimulus, awareness-related ROI) selectively correlated with visual awareness, whether the stimuli were attended or not. Third, a high-frequency component of the gamma-band response (76-90 Hz, 350-500 ms after stimulus, attention-related ROI) was modulated by spatial attention, whether the stimuli were consciously seen or not.

\section{Parametric analysis of the awareness- and attention-related activities}

To determine whether the contrastive approach used might have masked a more subtle interaction between visual awareness and spatial attention by averaging across repeated presentations of each condition, we performed a parametric analysis of the awareness- and attention-related activities at the single-trial level. This approach allowed us to assess the impact of the strong trialto-trial fluctuations of neural activity over the visual cortex on subjects' behavioral reports at the visual detection task (Arieli et al., 1996; Donner et al., 2007).

\section{Stimulus-present trials}

Trials from each subject were first sorted into four equally sized bins ( $n \approx 110$ trials per bin and per subject), from low to high power of oscillatory activity in the alpha $(10-12 \mathrm{~Hz}, 300-600 \mathrm{~ms}$ after cue, orienting-related ROI), awareness-related gamma (54-64 Hz, 240-500 ms after stimulus, awareness-related ROI), or attention-related gamma $(76-90 \mathrm{~Hz}, 350-500 \mathrm{~ms}$ after stimulus, attention-related ROI) frequency ranges. We then defined a detection index for each subject as the detection rate increase or decrease of each bin relative to the mean detection rate of the subject, so that positive (and negative) detection indices corresponded to many (and few) consciously seen stimuli in that bin, respectively (Linkenkaer-Hansen et al., 2004). Finally, we computed the Spearman's rank correlation coefficient between the power of oscillatory activity and the detection index across subjects. A null correlation coefficient indicates that neural and behavioral measures are unrelated, whereas a positive or negative correlation coefficient denotes a monotonic relationship between the two measures, i.e., a significant contribution of neural activity to the seen-unseen decision.

Decreases of posterior alpha-band activity during the orienting of spatial attention $(10-12 \mathrm{~Hz}, 300-600 \mathrm{~ms}$ after cue, orienting-related ROI) did not correlate with subjects' awareness of upcoming stimuli presented at the attended location (Spearman's rank correlation test, $\rho=-0.02, \mathrm{df}=46, p>0.5, \mathrm{NS}$ ) (Fig. $6 \mathrm{~A}$, left). This result further suggests that visual awareness did not merely reflect the orienting of spatial attention, even at the single-trial level.

In contrast, the awareness-related gamma-band activity (54-64 Hz, 240-500 ms after stimulus, awareness-related ROI) showed a positive correlation with subjects' behavioral reports of awareness (Spearman's rank correlation test, $\rho=+0.63$, df $=46$, $p<0.001$ ) (Fig. 6A, middle). A linear fit successfully accounted for this correlation (Pearson's linear regression test, $r=+0.67$, $\mathrm{df}=46, p<0.001)$. Each of the 12 subjects showed a positive correlation coefficient, thus confirming the robustness of the result. Furthermore, the regression slope between behavioral and neural measures was unaffected by spatial attention (general regression model, interaction: power $\times$ attention, $F_{(1,92)}=0.0, p>$ 


\section{Stimulus-present trials}

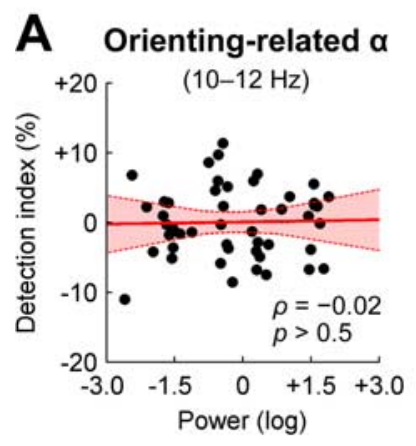

C

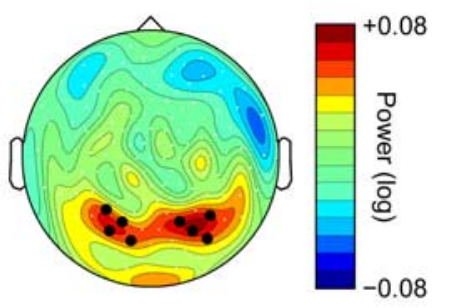

Awareness-related $\mathbf{Y}$

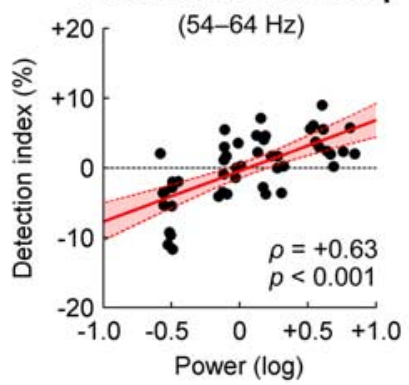

Stimulus-absent trials

D

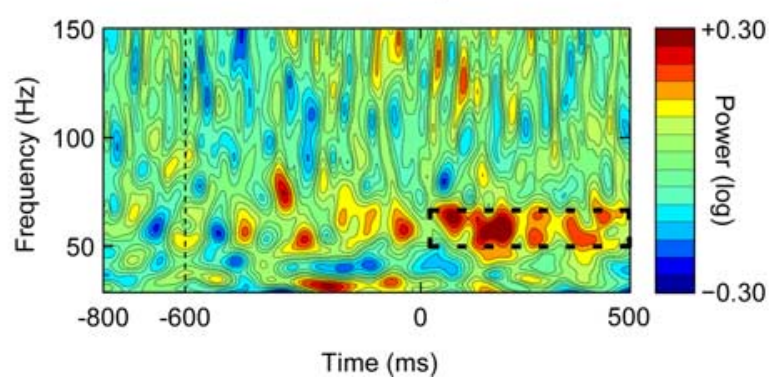

B
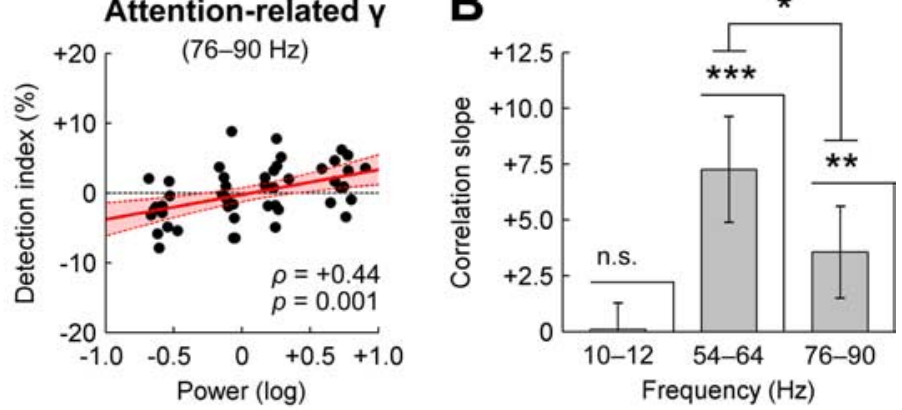

E Awareness-related y

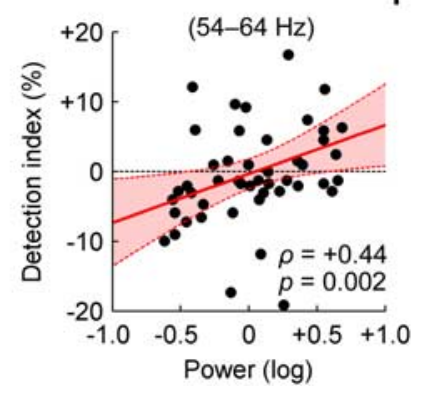

Figure 6. Parametric analysis of the awareness- and attention-related activities. Stimulus-present trials. $A$, The orienting-related alpha-band activity (10-12 Hz, $300-600 \mathrm{~ms}$ after cue) did not correlate with subjective reports of awareness (left). The awareness-related gamma-band activity ( $54-64 \mathrm{~Hz}, 240-500 \mathrm{~ms}$ after stimulus) positively correlated with subjective reports of awareness (middle). The attention-related gamma-band activity $(76-90 \mathrm{~Hz}, 350-500 \mathrm{~ms}$ after stimulus) also correlated with subjective reports of awareness (right). $\boldsymbol{B}$, The slope of the regression line (shown in red in $\boldsymbol{A}$ ) was smaller for the attention-related gamma-band activity than for the awareness-related gamma-band activity. Stimulus-absent trials. $\boldsymbol{C}$, Scalp topography of the mid-frequency gamma-band activity $(54-64 \mathrm{~Hz})$ in the absence of stimulus. Thick sensors indicate the peak of the response $(n=8)$. D, Time-frequency representation of the gamma-band activity for false-alarm reports over the group of sensors highlighted in $\boldsymbol{C}$, showing a selective increase of gamma-band activity in the mid-frequency range (dashed box) but not in the high-frequency range. $\boldsymbol{E}$, The mid-frequency gamma-band activity positively correlated with false-alarm reports, i.e., awareness of visually absent stimuli. Dashed red lines indicate the $95 \%$ confidence interval of the regression lines. The Spearman's rank correlation coefficients $\rho$ and their corresponding two-tailed $p$ values are shown. ${ }^{*} p<0.05$; ${ }^{* *} p<0.01$; ${ }^{* * *} p<0.001$; n.s., a nonsignificant effect.

Table 1. Correlation between trial-to-trial power fluctuations of awareness- and attention-related gamma-band activities at the single-subject level

\begin{tabular}{lllllllllllll}
\hline & 01 & 02 & 03 & 04 & 05 & 06 & 07 & 08 & 09 & 10 & 11 & 12 \\
\hline$n$ (active) & 544 & 469 & 432 & 532 & 412 & 319 & 344 & 341 & 388 & 393 & 381 & 504 \\
Spearman's $\rho$ & +0.03 & +0.04 & +0.06 & -0.04 & -0.01 & +0.03 & +0.03 & +0.04 & +0.02 & +0.05 & +0.09 & +0.00 \\
$p$ value & 0.46 & 0.34 & 0.22 & 0.39 & 0.81 & 0.59 & 0.61 & 0.42 & 0.66 & 0.36 & 0.08 & 0.94 \\
Significance & NS & NS & NS & NS & NS & NS & NS & NS & NS & NS & NS & NS \\
\hline
\end{tabular}

For subjects $01-12$, we computed the Spearman's rank correlation test between the trial-to-trial power fluctuations of the awareness-related mid-frequency gamma-band activity $(54-64 \mathrm{~Hz}, 240$ - $500 \mathrm{~ms}$ after stimulus, awareness-related ROI) and the attention-related high-frequency gamma-band activity $(76-90 \mathrm{~Hz}, 350-500 \mathrm{~ms}$ after stimulus, attention-related ROI). The number of active samples ( $n$ ), the Spearman's rank correlation coefficient ( $\rho$ ), the two-tailed $p$ value, and the corresponding significance level are shown for each subject. None of the 12 subjects showed a significant correlation between the power fluctuations of awareness- and attention-related gamma-band activities at the single-trial level (mean $\rho=+0.03$, d.f. $\approx 440$, mean $p=0.49$, NS).

0.5 , NS). Therefore, the awareness-related gamma-band activity significantly correlated with subjective reports of visual awareness but was not modulated by spatial attention.

Identified as a correlate of the attentional modulation of consciously seen and unseen stimuli, the attention-related gammaband activity $(76-90 \mathrm{~Hz}, 350-500 \mathrm{~ms}$ after stimulus, attentionrelated ROI) also correlated positively with subjects' behavioral reports of awareness (Spearman's rank correlation test, $\rho=$ $+0.44, \mathrm{df}=46, p=0.001$; Pearson's linear regression test, $r=$ $+0.46, \mathrm{df}=46, p=0.001$ ) (Fig. $6 \mathrm{~A}$, right). Eleven of the 12 subjects showed a positive correlation coefficient in this frequency band. However, the corresponding regression slope was significantly smaller than for the awareness-related response (general regression model, interaction: power $\times$ frequency, $F_{(1,92)}=5.7, p=0.02$ ) (Fig. $6 B$ ). This result indicates that the attention-related gamma-band activity also influenced the outcome of the seen- unseen decision, although to a smaller degree than the awareness-related activity.

Importantly, none of the 12 subjects showed a significantly positive correlation coefficient between the trial-to-trial fluctuations of the awareness- and attention-related gamma-band activities over their respective ROIs (Spearman's rank correlation test, mean $\rho=$ +0.03 , df $\approx 440$, mean $p=0.49$, NS) (Table 1). The awarenessrelated and attention-related gamma-band activities are thus unrelated to each other at the single-trial level but nevertheless appear to contribute cumulatively to subjective reports of visual experience.

Stimulus-absent trials

When no stimulus was presented, subjects sometimes reported the presence of a stimulus during the trial. To assess whether 
these erroneous (false-alarm) reports were motivated by fluctuations of neural activity over the visual cortex, we extended the parametric analysis of the mid-frequency gamma-band activity to the fewer trials in which no stimulus was presented $(n \approx 20$ trials per bin and per subject). If this activity corresponds to an awareness-related process, then its trial-to-trial fluctuations should influence the outcome of the seen-unseen decision even when no stimulus was presented during the trial.

First, we identified the MEG sensors showing the strongest increase of mid-frequency gamma-band activity $(54-64 \mathrm{~Hz})$ in stimulus-absent trials during the same time window as in stimulus-present trials (240-500 ms after stimulus): this activity peaked bilaterally over the visual cortex. As for stimulus-present trials, we extracted the group of $n=8$ bilateral occipital sensors showing a significant increase of gamma-band activity in the time-frequency window of interest using a nonparametric randomization test ( $p_{c}<0.05$ corrected for multiple comparisons across contiguous sensors; see Materials and Methods) (Fig. 6C). This group of active sensors mostly overlapped the responding ROI identified for stimulus-present trials but slightly differed from the awareness-related ROI. The time-frequency representation of the gamma-band activity at this group of sensors for false-alarm reports showed a selective increase of gamma-band activity in the mid-frequency (awareness-related) range but not in the high-frequency (attention-related) range (Fig. 6D).

The parametric analysis of the mid-frequency gamma-band activity in stimulus-absent trials showed a positive correlation with false-alarm reports, i.e., subjective awareness of visually absent stimuli (Spearman's rank correlation test, $\rho=+0.44$, $\mathrm{df}=$ $46, p=0.002$; Pearson's linear regression test, $r=+0.34, \mathrm{df}=46$, $p=0.02$ ) (Fig. $6 E$ ). On a subject-by-subject basis, 9 of the 12 subjects showed a positive correlation coefficient in this frequency band. Furthermore, the regression slope between behavioral and neural measures was found to be independent from whether a stimulus was presented or not during the trial (general regression model, interaction: power $\times$ stimulus, $F_{(1,92)}=0.0$, $p>0.5$, NS). Therefore, an increasing level of mid-frequency gamma-band activity over occipital sensors corresponded to an increasing probability of consciously seeing a stimulus, whether the stimulus in question was actually attended, unattended, or even visually absent.

\section{Discussion}

In the present study, we directly assessed the amount of overlap between the neural correlates of visual awareness and spatial attention. We engaged human subjects in a spatial cueing task in which the subjects attended toward or away from faint stimuli that were consciously seen only half of the time. The factorial analysis of stimulus-induced oscillatory brain activity revealed a neural dissociation between visual awareness and spatial attention at different frequencies in the gamma range. Consciously seen stimuli induced an increase of mid-frequency gamma-band activity over the contralateral visual cortex that was independent of the focus of spatial attention. The trial-to-trial fluctuations of this activity correlated with the content of subjective visual experience in both stimulus-present and stimulus-absent trials. Conversely, spatial attention selectively modulated high-frequency gamma-band activity over the contralateral visual cortex whether stimuli were consciously seen or not. Importantly, these awareness- and attention-related activities were independent of each other. Their parametric analysis, performed at the singletrial level, confirmed that the awareness-related activity drove the seen- unseen decision but further showed that the attention- related activity also influenced the decision, although to a much smaller degree.

\section{A neural dissociation between visual awareness from spatial attention}

The mid-frequency and high-frequency gamma-band activities, although temporally overlapping, were independent of each other (Makeig et al., 1999). First, they showed clearly distinct functional modulations: the mid-frequency gamma-band activity was increased by visual awareness but was not modulated by spatial attention, whereas the high-frequency gamma-band activity was increased by spatial attention in response to both consciously seen and unseen stimuli. Second, their topographies were distinct, suggesting different underlying neural sources. The fact that both activities peaked over parieto-occipital sensors contralateral to the stimulated hemifield further suggest that they may emerge from retinotopic visual areas (Hoogenboom et al., 2006). Third, their power fluctuations did not correlate with each other at the single-trial level. All these results point toward a neural dissociation between the mid-frequency and highfrequency components of the gamma-band response to the faint stimuli. But do these two neural activities actually correspond to awareness- and attention-related processes?

Assessing awareness is a notoriously difficult task. Traditionally, subjective and objective criteria have been used based on introspective reports and forced-choice decisions, respectively (Merikle et al., 2001; Snodgrass et al., 2004). Here we confronted the two approaches to assess subjects' awareness of the faint stimuli: we used a subjective criterion based on subjects' reports of their visual experience (detection task) along with an objective criterion based on subjects' ability to identify the stimulus (forced-choice orientation discrimination task). In our experiment, it turns out that subjective and objective thresholds of conscious perception converge to define two distinct cognitive states: an aware state in which stimuli were reported as present and correctly identified, and an unaware state in which stimuli were reported as absent and identified at chance level. In other words, visual awareness was assessed both subjectively and objectively: consequently, we could show that the mid-frequency gamma-band activity is likely to correspond to an awarenessrelated process, but neither to a simple response bias nor to a purely accuracy-related process unrelated to subjective visual experience (Hannula et al., 2005; Schurger et al., 2006). Furthermore, the trial-to-trial fluctuations of this activity not only correlated with visual awareness in stimulus-present trials but also correlated well with false-alarm reports in stimulus-absent trials over nearby MEG sensors. A plausible explanation is that the faint stimuli used here induce weak neural responses that are hardly distinguishable from the spontaneous pattern of ongoing oscillatory activity in early visual areas (Engel et al., 2001; Kenet et al., 2003; Buzsaki, 2006). The stimuli are thus reported as present if they induce sufficient perturbations of the spontaneous pattern of ongoing activity, whereas fluctuations of ongoing activity themselves can be mistakenly reported if they reach the level of activity usually induced by the faint stimuli (Arieli et al., 1996; Thompson and Schall, 1999; Shulman et al., 2001; Super et al., 2001; Ress and Heeger, 2003).

Besides, several results indicate that subjects consistently attended toward the cued location. First, they showed the typical shortening of reaction times at the orientation discrimination task for attended stimuli (Posner et al., 1980). Second, the orienting of spatial attention resulted in contralateral decreases of posterior alpha-band activity (Worden et al., 2000; Sauseng et al., 
2005; Thut et al., 2006; Medendorp et al., 2007). Third, the highfrequency gamma-band activity was increased by spatial attention during stimulus presentation, as observed previously in similar conditions using visual stimuli at maximal contrast (Gruber et al., 1999; Fries et al., 2001; Landau et al., 2007). This highfrequency oscillatory activity is therefore likely to correspond to an attention-related process (Womelsdorf and Fries, 2007). Moreover, the fact that this attentional modulation was present whether stimuli were consciously seen or not adds to the growing evidence that attentional processes can influence the processing of unseen stimuli (Naccache et al., 2002; Woodman and Luck, 2003; Kentridge et al., 2004; Sumner et al., 2006; Bahrami et al., 2007).

\section{Visual awareness and spatial attention have different time courses}

In the present study, the time courses of the awareness- and attention-related effects on the gamma-band response were quite different: whereas consciously seen and unseen trials diverged from $240 \mathrm{~ms}$ after stimulus onward, spatial attention started to modulate gamma-band activity at $350 \mathrm{~ms}$ after stimulus only. It would be tempting to relate this temporal succession to a causal relationship, with visual awareness driving spatial attention. However, we have shown that the awareness- and attentionrelated gamma-band activities were not coupled on a trial-pertrial basis: although the awareness- and attention-related effects showed up successively, they were both sustained and seemed to operate in parallel.

The attention-related effect reported here is quite late compared with other effects classically found in the literature: attentional modulations typically show up as early as $100 \mathrm{~ms}$ after stimulus, in human event-related potentials (Luck et al., 2000) or in gamma-band activity in monkey visual area V4 (Fries et al., 2001). A major difference between the present and other studies is that we used faint stimuli at threshold contrast $(\sim 3 \%)$ and not at maximal contrast $(>80 \%)$. The appearance of the faint stimuli triggered a detectable gamma-band response over the visual cortex but a weak one compared with the responses induced by stimuli at maximal contrast (Hoogenboom et al., 2006; Vidal et al., 2006; Siegel et al., 2007). Top-down attentional biases might take more time to modulate such a weak response.

In contrast, the awareness-related effect appeared at earlier latencies, $\sim 240 \mathrm{~ms}$ after stimulus, in retinotopic visual areas. Again, this might be related to the nature of the stimuli used: at threshold contrast, fluctuations of neural activity in early sensory areas have been shown previously to influence the subjective experience of faint stimuli (Ress and Heeger, 2003; LinkenkaerHansen et al., 2004). These fluctuations and the weak neural responses to the faint stimuli might interact from the earliest steps of visual processing (Arieli et al., 1996), progressively leading to a dissociation between consciously seen and unseen trials that becomes large enough to be detected only after $200 \mathrm{~ms}$ (Sergent et al., 2005; Schurger et al., 2006; Del Cul et al., 2007; Koivisto et al., 2007).

\section{Visual awareness and spatial attention contribute to subjective visual experience}

On the one hand, visual awareness and spatial attention appeared independent at the neural level, as shown by the factorial analysis of stimulus-induced oscillatory brain activity. On the other hand, however, the two functions seemed mutually dependent at the behavioral level: visual awareness was necessary for spatial attention to shorten reaction times, whereas spatial attention in- creased the proportion of consciously seen stimuli (Hawkins et al., 1990). How to explain this apparent discrepancy between behavioral and neural levels, particularly during the visual detection task?

We performed the parametric analysis of the awareness- and attention-related activities precisely to assess the respective influences of these activities on the seen-unseen decision. The results confirmed that the awareness-related activity drove the seenunseen decision but further revealed that the attention-related activity also partially correlated with the decision, in a much smaller and independent manner. One explanation is that the two activities do not directly influence each other but rather sum up to reach a perceptual threshold about the presence (or absence) of a faint stimulus during the trial. Such a mechanism could explain the higher proportion of consciously seen stimuli at the attended location without requiring a direct interaction between awareness- and attention-related processes (Koch, 2004). In other words, although visual awareness and spatial attention interact at the behavioral level (Carrasco et al., 2004), their neural correlates are here mostly independent during the visual processing of the faint stimuli and contribute cumulatively to subjective visual experience.

\section{Gamma-band activity reflects both visual awareness and spatial attention}

Importantly, the neural correlates of visual awareness and spatial attention identified in the present study were induced oscillations in the gamma range, proposed to reflect recurrent processing and neural communication at the population level (Singer, 1999; Tallon-Baudry and Bertrand, 1999; Fries, 2005). It should be noted here that other brain responses to the faint stimuli might contribute to subjective visual experience (Luck et al., 2000; Pins and Ffytche, 2003; Sergent et al., 2005; Del Cul et al., 2007; Melloni et al., 2007), but they could not be identified here because of the absence of a reliable evoked (i.e., time-locked) response.

Oscillatory activity in the gamma range has been functionally related, in separate studies, to conscious perception (Engel and Singer, 2001; Fries et al., 2002; Schurger et al., 2006; Wilke et al., 2006) as well as attentional selection (Gruber et al., 1999; Fries et al., 2001; Tallon-Baudry et al., 2005; Landau et al., 2007; Womelsdorf and Fries, 2007). Our results thus confirm the involvement of gamma-band activity in both visual awareness and spatial attention. However, the results further show that distinct frequency components of the gamma-band response may support flexibly and simultaneously distinct cognitive functions (Vidal et al., 2006; Buschman and Miller, 2007). Such a frequency specialization suggests that gamma-band synchronization is not a monolithic mechanism in charge of a single cognitive function (Tallon-Baudry, 2004; Tallon-Baudry et al., 2005; Buzsaki, 2006). In the present study, the modulation of the high-frequency gamma-band activity could reflect the selective neural amplification of attended signals in the visual cortex (Reynolds and Chelazzi, 2004), whereas the mid-frequency gamma-band activity could reflect the reverberation process that characterizes perceptual awareness per se according to current views (Tononi and Edelman, 1998; Dehaene et al., 2006; Lamme, 2006; Buzsaki, 2007).

\section{Distinct neural correlates imply distinct functional roles}

Because visual awareness and spatial attention can operate independently of each other at the neural level, the two should be considered as distinct mental operations supporting distinct brain functions (Lamme, 2003, 2004; Koch and Tsuchiya, 2007). 
If the role of spatial attention is thought to be the prioritization of visual processing via the selective amplification of neural signals in the visual cortex (Reynolds and Chelazzi, 2004; Womelsdorf and Fries, 2007), what could be the functional role of visual awareness? At the neural level, one possibility is that visual awareness supports the reverberation of sensory information in visual areas (Lamme, 2006), possibly to ensure the availability of this information to the rest of the brain (Haynes et al., 2005; Melloni et al., 2007), in particular prefrontal areas that allow for flexible cognitive control (Miller and Cohen, 2001; Dehaene et al., 2006). Therefore, although both visual awareness and spatial attention shape our subjective visual experience, the two can rely on distinct and independent brain processes: from a neural perspective, seeing something consciously appears to be different from attending to it.

\section{References}

Arieli A, Sterkin A, Grinvald A, Aertsen A (1996) Dynamics of ongoing activity: explanation of the large variability in evoked cortical responses. Science 273:1868-1871.

Babiloni C, Vecchio F, Miriello M, Romani GL, Rossini PM (2006) Visuospatial consciousness and parieto-occipital areas: a high-resolution EEG study. Cereb Cortex 16:37-46.

Bahrami B, Lavie N, Rees G (2007) Attentional load modulates responses of human primary visual cortex to invisible stimuli. Curr Biol 17:509-513.

Block N (1995) On a confusion about a function of consciousness. Behav Brain Sci 18:227-247.

Block N (2005) Two neural correlates of consciousness. Trends Cogn Sci 9:46-52.

Brainard DH (1997) The psychophysics toolbox. Spat Vis 10:433-436.

Buschman TJ, Miller EK (2007) Top-down versus bottom-up control of attention in the prefrontal and posterior parietal cortices. Science $315: 1860-1862$.

Buzsaki G (2006) Rhythms of the brain. Oxford, UK: Oxford UP.

Buzsaki G (2007) The structure of consciousness. Nature 446:267.

Carrasco M, Ling S, Read S (2004) Attention alters appearance. Nat Neurosci 7:308-313.

Dehaene S, Naccache L, Cohen L, Bihan DL, Mangin JF, Poline JB, Riviere D (2001) Cerebral mechanisms of word masking and unconscious repetition priming. Nat Neurosci 4:752-758.

Dehaene S, Changeux JP, Naccache L, Sackur J, Sergent C (2006) Conscious, preconscious, and subliminal processing: a testable taxonomy. Trends Cogn Sci 10:204-211.

Del Cul A, Baillet S, Dehaene S (2007) Brain dynamics underlying the nonlinear threshold for access to consciousness. PLoS Biol 5:e260.

Dobbins IG, Schnyer DM, Verfaellie M, Schacter DL (2004) Cortical activity reductions during repetition priming can result from rapid response learning. Nature 428:316-319.

Donner TH, Siegel M, Oostenveld R, Fries P, Bauer M, Engel AK (2007) Population activity in the human dorsal pathway predicts the accuracy of visual motion detection. J Neurophysiol 98:345-359.

Engel AK, Singer W (2001) Temporal binding and the neural correlates of sensory awareness. Trends Cogn Sci 5:16-25.

Engel AK, Fries P, Singer W (2001) Dynamic predictions: oscillations and synchrony in top-down processing. Nat Rev Neurosci 2:704-716.

Fernandez-Duque D, Grossi G, Thornton IM, Neville HJ (2003) Representation of change: separate electrophysiological markers of attention, awareness, and implicit processing. J Cogn Neurosci 15:491-507.

Fries P (2005) A mechanism for cognitive dynamics: neuronal communication through neuronal coherence. Trends Cogn Sci 9:474-480.

Fries P, Reynolds JH, Rorie AE, Desimone R (2001) Modulation of oscillatory neuronal synchronization by selective visual attention. Science 291:1560-1563.

Fries P, Schroder JH, Roelfsema PR, Singer W, Engel AK (2002) Oscillatory neuronal synchronization in primary visual cortex as a correlate of stimulus selection. J Neurosci 22:3739-3754.

Fries P, Nikolic D, Singer W (2007) The gamma cycle. Trends Neurosci 30:309-316.

Green DM, Swets JA (1966) Signal detection theory and psychophysics. New York: Wiley.
Gruber T, Muller MM, Keil A, Elbert T (1999) Selective visual-spatial attention alters induced gamma band responses in the human EEG. Clin Neurophysiol 110:2074-2085.

Hannula DE, Simons DJ, Cohen NJ (2005) Imaging implicit perception: promise and pitfalls. Nat Rev Neurosci 6:247-255.

Hawkins HL, Hillyard SA, Luck SJ, Mouloua M, Downing CJ, Woodward DP (1990) Visual attention modulates signal detectability. J Exp Psychol Hum Percept Perform 16:802-811.

Haynes JD, Driver J, Rees G (2005) Visibility reflects dynamic changes of effective connectivity between V1 and fusiform cortex. Neuron 46:811-821.

Hoogenboom N, Schoffelen JM, Oostenveld R, Parkes LM, Fries P (2006) Localizing human visual gamma-band activity in frequency, time and space. NeuroImage 29:764-773.

James W (1890) Principles of psychology. New York: Henry Holt.

Jokisch D, Jensen O (2007) Modulation of gamma and alpha activity during a working memory task engaging the dorsal or ventral stream. J Neurosci 27:3244-3251.

Kenet T, Bibitchkov D, Tsodyks M, Grinvald A, Arieli A (2003) Spontaneously emerging cortical representations of visual attributes. Nature 425:954-956.

Kentridge RW, Heywood CA, Weiskrantz L (2004) Spatial attention speeds discrimination without awareness in blindsight. Neuropsychologia 42:831-835.

Koch C (2004) The quest for consciousness: a neurobiological approach. Englewood, CO: Roberts and Company Publishers.

Koch C, Tsuchiya N (2007) Attention and consciousness: two distinct brain processes. Trends Cogn Sci 11:16-22.

Koivisto M, Revonsuo A (2007) Electrophysiological correlates of visual consciousness and selective attention. NeuroReport 18:753-756.

Koivisto M, Lahteenmaki M, Sorensen TA, Vangkilde S, Overgaard M, Revonsuo A (2007) The earliest electrophysiological correlate of visual awareness? Brain Cogn 66:91-103.

Lamme VA (2003) Why visual attention and awareness are different. Trends Cogn Sci 7:12-18.

Lamme VA (2004) Separate neural definitions of visual consciousness and visual attention; a case for phenomenal awareness. Neural Netw 17:861-872.

Lamme VA (2006) Towards a true neural stance on consciousness. Trends Cogn Sci 10:494-501.

Landau AN, Esterman M, Robertson LC, Bentin S, Prinzmetal W (2007) Different effects of voluntary and involuntary attention on EEG activity in the gamma band. J Neurosci 27:11986-11990.

Linkenkaer-Hansen K, Nikulin VV, Palva S, Ilmoniemi RJ, Palva JM (2004) Prestimulus oscillations enhance psychophysical performance in humans. J Neurosci 24:10186-10190.

Luck SJ, Woodman GF, Vogel EK (2000) Event-related potential studies of attention. Trends Cogn Sci 4:432-440.

Macmillan NA, Creelman CD (2005) Detection theory: a user's guide, Ed 2. London: Erlbaum Associates.

Makeig S, Westerfield M, Jung TP, Covington J, Townsend J, Sejnowski TJ, Courchesne E (1999) Functionally independent components of the late positive event-related potential during visual spatial attention. J Neurosci 19:2665-2680.

Medendorp WP, Kramer GF, Jensen O, Oostenveld R, Schoffelen JM, Fries P (2007) Oscillatory activity in human parietal and occipital cortex shows hemispheric lateralization and memory effects in a delayed double-step saccade task. Cereb Cortex 17:2364-2374.

Melloni L, Molina C, Pena M, Torres D, Singer W, Rodriguez E (2007) Synchronization of neural activity across cortical areas correlates with conscious perception. J Neurosci 27:2858-2865.

Merikle PM, Smilek D, Eastwood JD (2001) Perception without awareness: perspectives from cognitive psychology. Cognition 79:115-134.

Metzinger T, ed (2000) Neural correlates of consciousness: empirical and conceptual questions. Cambridge, MA: MIT.

Miller EK, Cohen JD (2001) An integrative theory of prefrontal cortex function. Annu Rev Neurosci 24:167-202.

Naccache L, Blandin E, Dehaene S (2002) Unconscious masked priming depends on temporal attention. Psychol Sci 13:416-424.

Nichols TE, Holmes AP (2002) Nonparametric permutation tests for functional neuroimaging: a primer with examples. Hum Brain Mapp 15:1-25. 
Pelli DG (1997) The VideoToolbox software for visual psychophysics: transforming numbers into movies. Spat Vis 10:437-442.

Pins D, Ffytche D (2003) The neural correlates of conscious vision. Cereb Cortex 13:461-474.

Portin K, Vanni S, Virsu V, Hari R (1999) Stronger occipital cortical activation to lower than upper visual field stimuli. Neuromagnetic recordings. Exp Brain Res 124:287-294.

Posner MI (1980) Orienting of attention. Q J Exp Psychol 32:3-25.

Posner MI, Snyder CR, Davidson BJ (1980) Attention and the detection of signals. J Exp Psychol 109:160-174.

Rees G, Kreiman G, Koch C (2002) Neural correlates of consciousness in humans. Nat Rev Neurosci 3:261-270.

Ress D, Heeger DJ (2003) Neuronal correlates of perception in early visual cortex. Nat Neurosci 6:414-420.

Reynolds JH, Chelazzi L (2004) Attentional modulation of visual processing. Annu Rev Neurosci 27:611-647.

Sauseng P, Klimesch W, Stadler W, Schabus M, Doppelmayr M, Hanslmayr S, Gruber WR, Birbaumer N (2005) A shift of visual spatial attention is selectively associated with human EEG alpha activity. Eur J Neurosci 22:2917-2926.

Schurger A, Cowey A, Tallon-Baudry C (2006) Induced gamma-band oscillations correlate with awareness in hemianopic patient GY. Neuropsychologia 44:1796-1803.

Searle JR (1992) The rediscovery of the mind. Cambridge, MA: MIT.

Sergent C, Baillet S, Dehaene S (2005) Timing of the brain events underlying access to consciousness during the attentional blink. Nat Neurosci 8:1391-1400.

Shulman GL, Ollinger JM, Linenweber M, Petersen SE, Corbetta M (2001) Multiple neural correlates of detection in the human brain. Proc Natl Acad Sci USA 98:313-318.

Siegel M, Donner TH, Oostenveld R, Fries P, Engel AK (2007) Highfrequency activity in human visual cortex is modulated by visual motion strength. Cereb Cortex 17:732-741.

Singer W (1999) Neuronal synchrony: a versatile code for the definition of relations? Neuron 24:49-65, 111-125.

Singer W, Gray CM (1995) Visual feature integration and the temporal correlation hypothesis. Annu Rev Neurosci 18:555-586.

Snodgrass M, Bernat E, Shevrin H (2004) Unconscious perception: a model-based approach to method and evidence. Percept Psychophys $66: 846-867$.
Sumner P, Tsai PC, Yu K, Nachev P (2006) Attentional modulation of sensorimotor processes in the absence of perceptual awareness. Proc Natl Acad Sci USA 103:10520-10525.

Super H, Spekreijse H, Lamme VA (2001) Two distinct modes of sensory processing observed in monkey primary visual cortex (V1). Nat Neurosci 4:304-310.

Tallon-Baudry C (2004) Attention and awareness in synchrony. Trends Cogn Sci 8:523-525.

Tallon-Baudry C, Bertrand O (1999) Oscillatory gamma activity in humans and its role in object representation. Trends Cogn Sci 3:151-162.

Tallon-Baudry C, Bertrand O, Henaff MA, Isnard J, Fischer C (2005) Attention modulates gamma-band oscillations differently in the human lateral occipital cortex and fusiform gyrus. Cereb Cortex 15:654-662.

Thompson KG, Schall JD (1999) The detection of visual signals by macaque frontal eye field during masking. Nat Neurosci 2:283-288.

Thut G, Nietzel A, Brandt SA, Pascual-Leone A (2006) Alpha-band electroencephalographic activity over occipital cortex indexes visuospatial attention bias and predicts visual target detection. J Neurosci 26:9494-9502.

Tononi G, Edelman GM (1998) Consciousness and complexity. Science 282:1846-1851.

Tyler CW (1997) Colour bit-stealing to enhance the luminance resolution of digital displays on a single pixel basis. Spat Vis 10:369-377.

Vidal JR, Chaumon M, O’Regan JK, Tallon-Baudry C (2006) Visual grouping and the focusing of attention induce gamma-band oscillations at different frequencies in human magnetoencephalogram signals. J Cogn Neurosci 18:1850-1862.

Wilke M, Logothetis NK, Leopold DA (2006) Local field potential reflects perceptual suppression in monkey visual cortex. Proc Natl Acad Sci USA 103:17507-17512.

Womelsdorf T, Fries P (2007) The role of neuronal synchronization in selective attention. Curr Opin Neurobiol 17:154-160.

Woodman GF, Luck SJ (2003) Dissociations among attention, perception, and awareness during object-substitution masking. Psychol Sci 14:605-611.

Worden MS, Foxe JJ, Wang N, Simpson GV (2000) Anticipatory biasing of visuospatial attention indexed by retinotopically specific alpha-band electroencephalography increases over occipital cortex. J Neurosci 20: RC63(1-6). 